\title{
RiboTag-Seq Reveals the Activation of the Unfolded Protein Response in Striatal Microglia Induced by Ethanol Withdrawal
}

\author{
Brett D. Dufour ${ }^{5}$, Kevin R. Coffey ${ }^{1}$, Atom J. Lesiak ${ }^{3}$, Gwenn A. Garden ${ }^{4}$, and John F. Neumaier ${ }^{1,2 *}$ \\ Departments of Psychiatry \& Behavioral Sciences ${ }^{1}$ and Pharmacology², University of Washington School of Medicine, Seattle, WA, 98104, USA; \\ Department of Genome Sciences ${ }^{3}$, University of Washington School of Medicine, Seattle, WA, 98104, USA; \\ Department of Neurology4, University of North Carolina Chapel Hill, NC, 27599, USA; \\ Department of Pathology and Laboratory Medicine ${ }^{5}$, University of California Davis, CA, 95616, USA \\ *Corresponding Author: John F. Neumaier, MD, PhD; Phone: 206-897-5803; Email: neumaier@uw.edu
}

Repeated cycles of alcohol intoxication and withdrawal both induce profound changes in gene expression that can contribute to the physiological and behavioral consequences of ethanol. Since neuroinflammation is an important consequence of these changes, we used a novel strategy to investigate the impact of repeated cycles of chronic intermittent ethanol vapor and withdrawal on the RNAs actively undergoing translation in striatal microglia. RiboTag was selectively expressed in the microglia of transgenic mice and was used to immunopurify the RNA "translatome" from striatal microglia, yielding a snapshot of RNA translation during alcohol intoxication and after 8 hours of withdrawal. We obtained highly enriched microglial RNAs and analyzed these in individual animals by deep sequencing. We found a dramatic shift in gene expression during acute intoxication compared to air-exposed controls, with increases in genes and pathways associated with cytokine signaling, indicating increased neuroinflammation and microglial activation. After 8 hours of ethanol withdrawal, many inflammatory pathways remained upregulated but phagocytotic and proapoptotic pathways were increased. Using an unbiased bioinformatic method, weighted gene coexpression network analysis, multiple differentially expressed gene modules were identified. One in particular was differentially expressed in ethanol intoxicated vs. withdrawing animals, and there was a strong correlation between the centrality of the genes to this gene network and their individual statistical significance in differential expression. The unfolded protein response was over-represented in this network after withdrawal. The induction of this pathway in microglia is important since this cellular stress response can either lead towards restoration of normal function or apoptosis.

Ethanol | Withdrawal | Microglia | RiboTag-Seq | WGCNA

Alcohol use disorder (AUD) is prevalent, occurring in $5.8 \%$ of adults in the U.S. ${ }^{1}$ at an estimated annual cost of $\$ 249$ billion annually ${ }^{2}$. The discontinuation of alcohol use in dependent individuals results in severe and persistent negative health effects. Acute withdrawal syndrome produces intense physiological and psychological distress and can lead to delirium tremens $^{3}$, seizures ${ }^{4}$, and even death ${ }^{5}$. Long term withdrawal/abstinence often results in persistent anxiety and mood disturbance that can last for months in alcohol-dependent individuals ${ }^{6}$, which contributes to the propensity for relapse by way of negative reinforcement ${ }^{7}$.

Chronic AUD is also associated with neurotoxicity, neuroinflammation, and immune activation (reviewed extensively in ${ }^{8}$ ). Acute alcohol use leads to two waves of toxicity - alcohol metabolism during acute intoxication produces toxic byproducts, including aldehydes and reactive oxygen species ${ }^{9}$, and acute withdrawal also leads to CNS hyperexcitability due to reduced GABAergic tone ${ }^{10}$ and enhanced glutamatergic activity ${ }^{11}$. In individuals with AUD, the degree of toxicity is exacerbated both by dose and duration of intake ${ }^{12,13}$. Furthermore, repeated attempts at alcohol cessation in dependent individuals is common before achieving sustained abstinence, and a kindling model has been proposed to explain how these repeated cycles of alcohol use and withdrawal lead to successive increases in neuroinflammation, neurotoxicity, neurodegeneration, and withdrawal symptom severity ${ }^{14}$. Indeed, individuals with a history of high levels of chronic alcohol use show significant levels of neuronal cell loss ${ }^{12,13}$, astrocyte and oligodendrocyte pathology ${ }^{13}$, microglial activation ${ }^{15}$ and increased neuroimmune signaling ${ }^{15}$. While there is a long-standing rationale for how alcohol induced toxicity to neurons may further contribute to AUD (e.g. neuron loss in the orbitofrontal cortex likely contributes to deficits in inhibitory control ${ }^{13,16}$ ) and alcohol withdrawal symptomology,

Author Contributions. Brett Dufour performed the experiment, analyzed the data, and wrote the manuscript. Kevin Coffey performed the experiment, analyzed the data, and wrote the manuscript. Atom Lesiak performed the experiment. Gwenn Garden provided animals, assisted in data interpretation, and wrote the manuscript. John Neumaier designed the experiment and wrote the manuscript

Code and Data Availability. All RNA-Seq files and the RNA-Seq pipeline are available on our Galaxy Server. All code and data used for figure generation are available in our Github Repository. The topological overlap Matrices are also hosted on the Galaxy server because of file size limitations associated with GitHub.

Acknowledgments. Supported by R21 DA044757 and T32 DA007278.

Competing Interests. The authors have no conflicts of interest to disclose. 

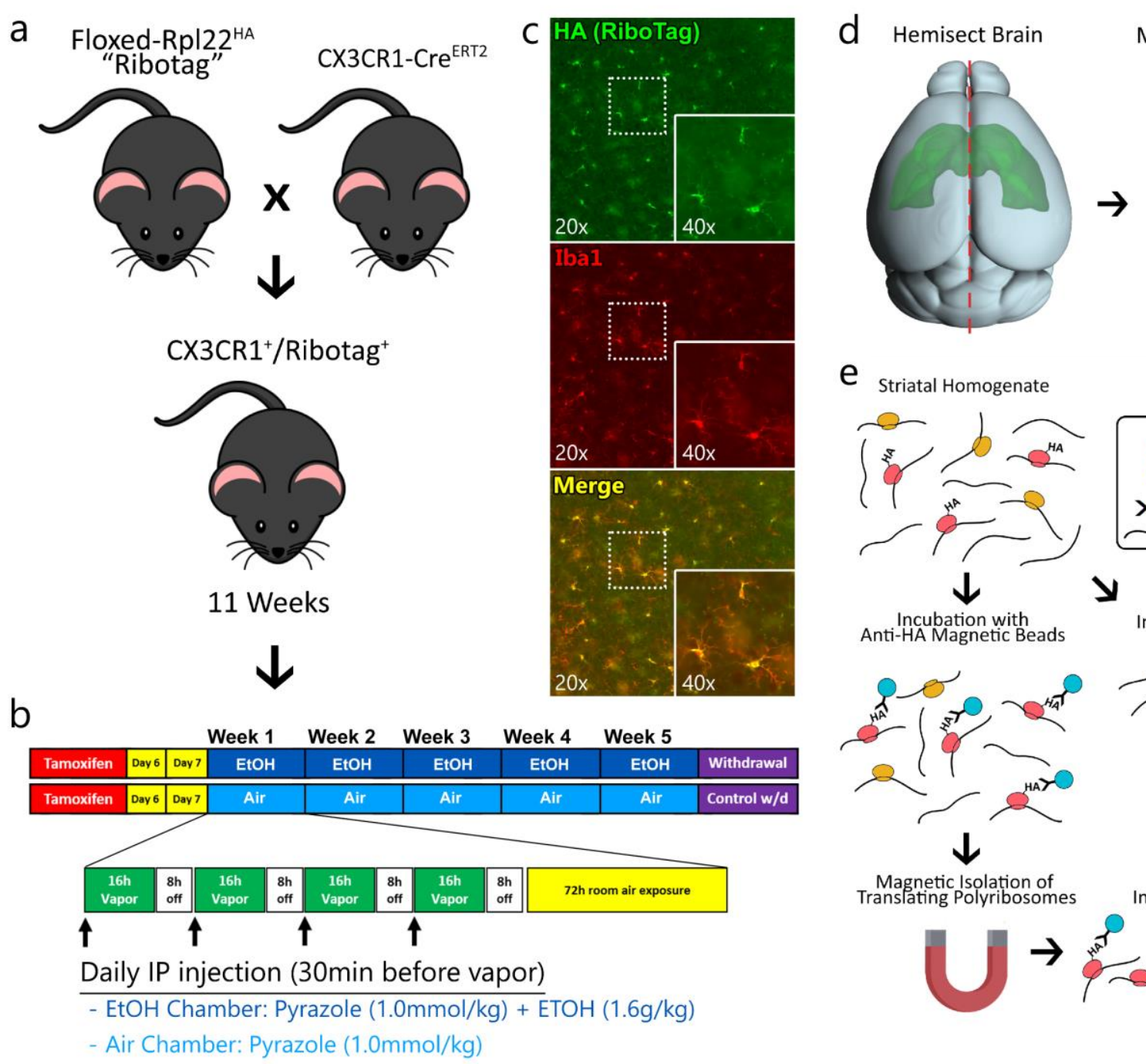

Microdisect Striatum

e

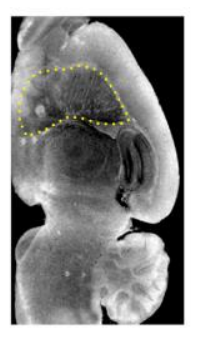

Striatal Homogenate
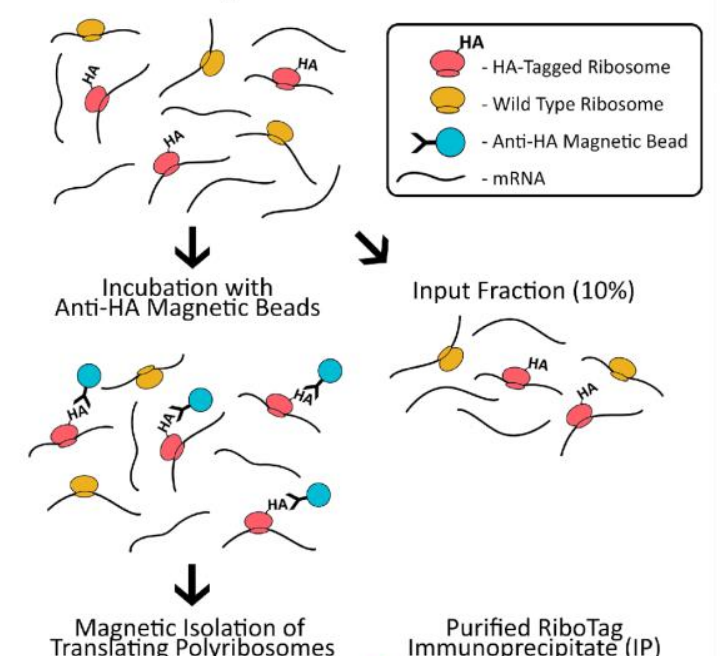

Translating Polyribosomes

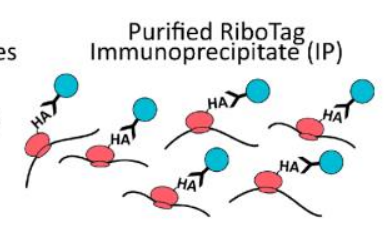

Figure 1. Experimental Overview | a, Experimental male and female mice were generated by crossing tamoxifen-inducible CX3CR1-CreERT2 hemizygous mice with homozygous floxed RiboTag mice. $\mathbf{b}$, The experiment was performed as a $2 X 2$ treatment design ( $n=6 / g r o u p$ ) with all mice receiving tamoxifen for 5 days at 14 weeks. At $\sim 15$ weeks of age the mice underwent chronic intermittent ethanol exposure (or air exposure). Animals were sacrificed after the week- 5 session at either 0 hours or 8 hours after the final exposure. c, Brains from these animals express HAtagged ribosomes exclusively in microglia. $d$, The brains were rapidly extracted, hemisected, and the striatum was microdissected and homogenized. d, RiboTag isolation procedure involves incubating tissue samples with anti-hemagglutinin antibody magnetic beads and performing magnetic isolation of RiboTag-positive, ribosome bound RNAs.

the contributions of alcohol associated glial pathology and neuroimmune activation to AUD and alcohol withdrawal symptomology are less clear.

As the primary resident immune cells of the brain, microglia are key regulators in neuroinflammation. At 'rest', they actively surveil the brain for alterations in homeostasis detected by numerous membrane embedded receptors ${ }^{17,18}$. Thus, microglia play a crucial role in healthy brain function in diverse manners, including modulating neurogenesis, neuronal circuit formation, synapse maturation during neurodevelopment, and by regulating neuronal signaling and neuroplasticity in the healthy adult brain 19. However, in cases of tissue trauma microglia will "activate" migrating to the site of trauma, proliferate, change their morphology (increased soma and shorter processes) and regulate the neuroinflammatory response by secreting neuroimmune and trophic factors and by phagocytosing debris ${ }^{17}$. In the case of neurotoxicity, microglia not only modulate the inflammatory response through cytokine and chemokine release ${ }^{17,18}$, they can also directly regulate cell survival by providing trophic support, or act as executioner by inducing apoptosis in aberrant cells ${ }^{17}$. The transition from resting to activated states is clearly more complex than a simple switch and involves many adaptations in microglial gene expression that may have important implications for the role of microglia in AUDs.

Alcohol use and withdrawal have profound effects of microglial function, and these alcohol-induced alterations in microglial function in turn contribute to alcohol associated behaviors and withdrawal symptomology ${ }^{8}$. However, many questions remain regarding how microglia respond to alcohol on 

available under aCC-BY-NC-ND 4.0 International license.

a molecular level; including which changes are adaptive and which exacerbate pathology, and how these microglial alterations in turn impact alcohol induced neurotoxicity, physiological withdrawal symptoms, and behavioral consequences such as craving and impulsivity. In the series of experiments presented here, we used a bioinformatics approach to characterize the effects of alcohol intoxication and withdrawal on microglia, by assessing alterations in microglia-specific gene expression. To do so, we utilized transgenic mice expressing RiboTag selectively in microglia, thus enabling specific isolation of actively translating mRNA transcripts from striatum. Since Chronic Intermittent Ethanol (CIE) primes and activates microglia and induces potent neuroinflammation in exposed subjects ${ }^{8}$, we used the well-established vapor CIE paradigm to identify the engagement of alcohol-sensitive, microglia-specific molecular pathways. We identified candidate molecular targets for potential therapeutic intervention. In particular, we detected evidence for an early and dramatic induction of the unfolded protein response, a cellular stress response that contends with misfolded proteins that impair cellular health, at a timepoint prior to the induction of typical morphological features of microglial activation, suggesting that this process may play a pivotal pathological role.

\section{Methods}

Subjects. Experimental subjects were generated by crossing hemizygous Cx3CR1-Cre breeder mice (B6.129P2(C)CX3Cr1 ${ }^{\text {tm2.1(cre/ERT2)Jung } / J, ~ S t o c k ~ \# 020940, ~ T h e ~ J a c k s o n ~ L a b o r a t o r y, ~}$ ME) with homozygous Ribotag breeder mice (B6J.129(Cg)-Rp/22 tm1.1Psam/SjJ, Stock \# 029977, The Jackson Laboratory, ME). Cx3Cr1-Cre/Ribotag double transgenic mice (Figure 1a) were utilized for RNA-Seq experiments to characterize the effect of ethanol intoxication and withdrawal on the microglial translatome. The Ribotag ( $\mathrm{C} \times 3 \mathrm{Cr} 1-\mathrm{Cre}$ null) single transgenic littermates were utilized for histology and blood collection. Mice were group housed with same sex littermates (up to 5 per cage) and provided with ad-libitum food and water and a 14-10 lightdark cycle. All procedures were approved by the University of Washington Institutional Animal Care and Use Committee, and consistent with ethical guidelines in the Guide ${ }^{20}$.

Tamoxifen Induction of CRE. Four weeks before the start of the Chronic Intermittent Ethanol (CIE) paradigm, Cx3Cr1-Cre/Ribotag mice were injected IP with $75 \mathrm{mg} / \mathrm{kg}$ Tamoxifen $(20 \mathrm{mg} / \mathrm{mL}$ tamoxifen in corn oil) once daily for five consecutive days at 14 weeks of age. Microglia specific Ribotag expression was confirmed in a subset of subjects using double label immunofluorescence for IBA-1 and HA (Figure 1b). Two additional $\mathrm{C} \times 3 \mathrm{Cr} 1-\mathrm{Cre}-\mathrm{Ribotag}$ mice were injected with only vehicle (corn oil) over 5 days, also underwent the CIE paradigm,
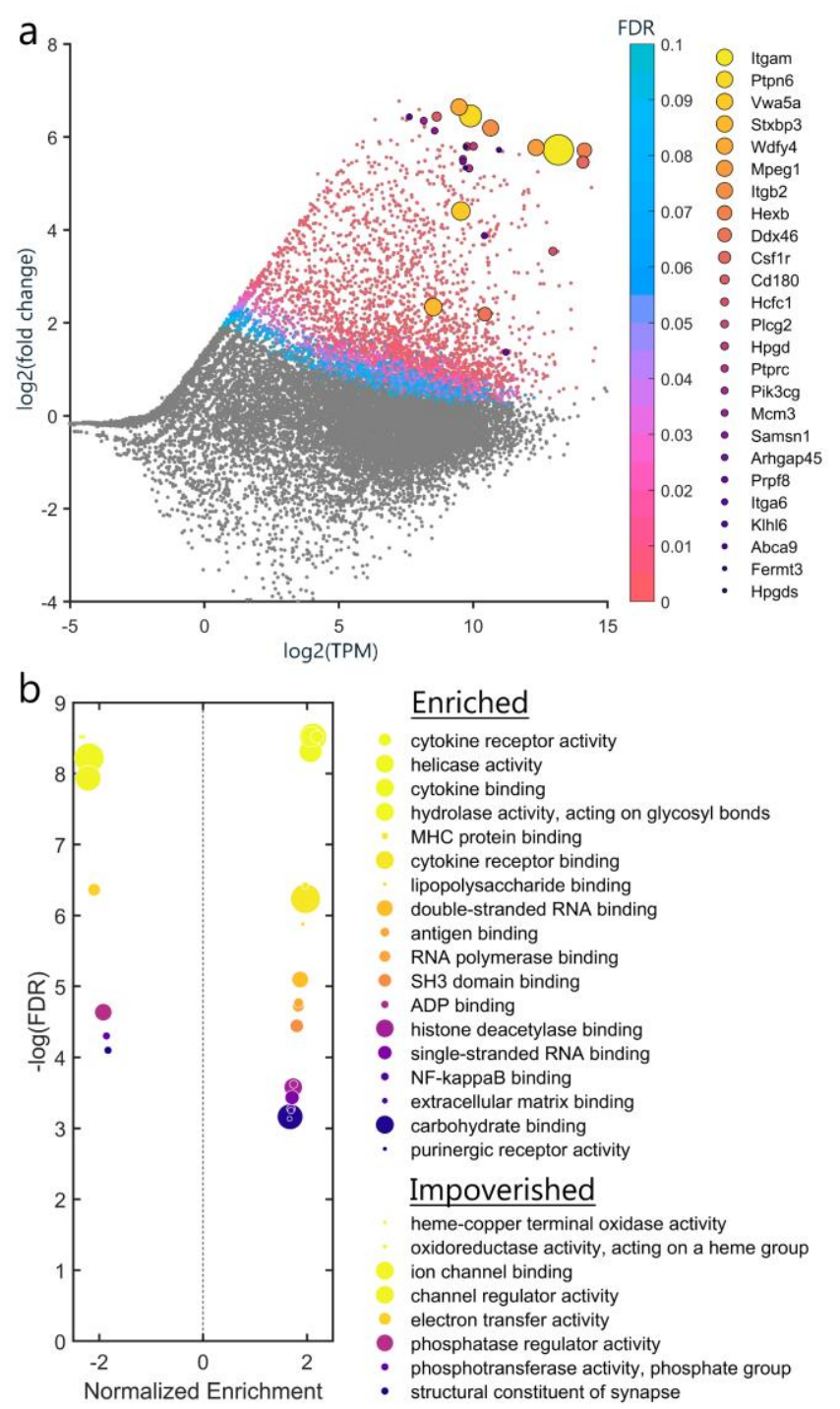

Enriched

cytokine receptor activity

helicase activity

cytokine binding

hydrolase activity, acting on glycosyl bonds

MHC protein binding

cytokine receptor binding

lipopolysaccharide binding

double-stranded RNA binding

antigen binding

RNA polymerase binding

- SH3 domain binding

- ADP binding

histone deacetylase binding

- single-stranded RNA binding

- NF-kappaB binding

- extracellular matrix binding

- carbohydrate binding

- purinergic receptor activity

Impoverished

heme-copper terminal oxidase activity

oxidoreductase activity, acting on a heme group

ion channel binding

channel regulator activity

electron transfer activity

- phosphatase regulator activity

- phosphotransferase activity, phosphate group

- structural constituent of synapse

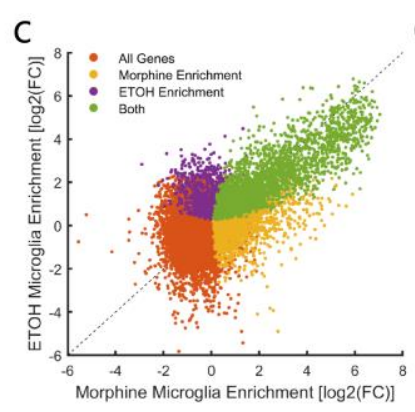

d

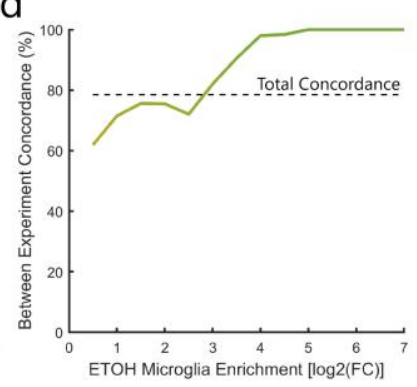

Figure 2. Ribotag Enrichment Is Consistant Across Studys I a, The RiboTag-Seq samples were dramatically enriched with microglial markers such as Itgam (cd11b) and CX3CR1. b, GSEA reveals an upregulation of genes associated with classical microglial gene sets such as "cytokine binding" and "purinergic receptor activity". c, 80\% of the differentially enriched genes in the present study were differentially expressed in our previous CX3CR1-Ribotag study assessing microglial changes in gene expression during morphine tolerance and withdrawal ${ }^{31}$. d, Genes that were highly enriched $(\log 2(\mathrm{fc})>4)$ shared nearly $100 \%$ concordance with our previous CX3CR1-Ribotag RNA-Seq Study. 

available under aCC-BY-NC-ND 4.0 International license.

and were used as Tamoxifen-negative controls for the RNA-Seq experiments.

Chronic Intermittent Ethanol Paradigm. Starting at 18 weeks of age, subjects underwent 5 consecutive weeks of Ethanol Vapor or Air exposure following the CIE paradigm (Figure 1b) ${ }^{21}$. Each week consisted of $4 \mathrm{CIE}$ sessions where subjects were exposed to 16 hours of ethanol vapor (or air), from $6 \mathrm{pm}$ to $10 \mathrm{am}$ the following day. After every $4 \mathrm{CIE}$ exposures, there was an 3 day break before starting the next week of sessions. 30 minutes before the start of each session, subjects were pretreated with an IP injection of either pyrazole alone $(1 \mathrm{mmol} / \mathrm{kg}$ bodyweight, in $0.9 \%$ saline) for Air treated subjects, or pyrazole $(1 \mathrm{mmol} / \mathrm{kg})+$ ethanol (1.6g/kg; $10 \%$ ethanol in $0.9 \%$ saline) for Ethanol Vapor treated subjects. The ethanol vapor concentration in chambers was $12 \mathrm{mg} / \mathrm{L}$ in order to consistently achieve sustained blood ethanol concentrations at approximately $200 \mathrm{mg} / \mathrm{dL}$ in pyrazole/ethanol pretreated subjects (Supplementary Figure 2). Food and water were available ad libitum during all sessions, including food at the bottom of the cage to enable feeding even when ataxic. Following every vapor exposure session, vapor exposed bedding and food was disposed and replaced with fresh bedding and food.

Two chambers were utilized, one for ethanol vapor treatment and the other for air control treatment, each with the dimensions of 32 " W $\times 14^{\prime \prime} \mathrm{H} \times 21.5^{\prime \prime} \mathrm{D}$. Total Air flow (40 SCFH) was matched between the two chambers during each session, and consistent across all sessions ${ }^{22}$. For the ethanol chamber, ambient air was bubbled through a side-arm flask containing $90 \%$ ethanol, producing ethanol vapors that were pumped into the chamber, and mixed with atmospheric air to achieve an ethanol vapor concentration of $12 \mathrm{mg} / \mathrm{L}$. Ethanol vapor concentration was measured using a breathalyzer (FC10; Lifeloc Technologies Inc.; Wheat Ridge, CO). Air pumps were turned on for 30 minutes before the start of each CIE session to confirm the ethanol vapor concentration. Excess air/vapor was ventilated into the rooms air exhaust intake.

Necropsy and Tissue Processing. All mice utilized for RNA-Seq analysis were sacrificed by decapitation, brains were quickly dissected and then bisected into left and right hemispheres. Whole striatum from the right hemisphere was microdissected, placed in $4^{\circ} \mathrm{C}$ supplemented Homogenization buffer ( $50 \mathrm{mM}$ Tris$\mathrm{HCl}, 100 \mathrm{mM} \mathrm{KCl}, 12 \mathrm{mM} \mathrm{MgCl}$, 1\% NP40, $1 \mathrm{mM} \mathrm{DTT,} 1 \times$ Protease inhibitor cocktail (Sigma-Aldrich), $200 \mathrm{U} / \mathrm{mL}$ RNasin (Promega, Madison, WI), $100 \mathrm{\mu g} / \mathrm{mL}$ cyclohexamide (SigmaAldrich), $1 \mathrm{mg} / \mathrm{mL}$ heparin (APP Pharmaceuticals, Lake Zurich, IL)), and immediately homogenized. Homogenates were centrifuged at $10 \mathrm{k}$ RPM for $10 \mathrm{~min}$, the supernatant collected and stored at $80^{\circ} \mathrm{C}$ until processed further for RNA extraction. The left hemisphere was snap frozen and stored at $-80^{\circ} \mathrm{C}$.
RiboTag RNA Isolation and Quantification. Striatal dissections homogenized in the supplemented homogenization buffer were processed using our standard RiboTag procedure described in detail in Lesiak et al., $2020^{23}$.

RNA-Seq Library Preparation and qPCR. RNA-Seq libraries were prepared using SMARTer Stranded Total RNA-Seq Kit v2 - Pico Input Mammalian (Takara Bio USA, Inc. Cat. 635007, Mountain View, CA). 10ng of RNA or average equivalent volumes of "no Tamoxifen" control samples were used to generate the libraries. RNA-Seq libraries were submitted to Northwest Genomics Center at University of Washington (Seattle, WA) where library quality control was measured using a BioAnalyzer, library concentrations were measured using Qubit dsDNA HS Assay Kit (ThermoFisher). Samples were normalized and pooled prior to cluster generation on HiSeq High Output for paired-end reads. RNA-Seq libraries were sequenced on the HiSeq4000, Paired-end 75bp with PhiX spike-in controls (7\%) (Illumina San Diego, CA).

Transcript Quantification and Quality Control. Raw fastq files were processed using multiple tools through the Galaxy platform ${ }^{24}$. Fastq files were inspected for quality using FastQC (Galaxy Version 0.7.0), and then passed to Salmon ${ }^{25}$ (Galaxy Version 0.8.2) for quantification of transcripts. The Salmon index was built using the protein coding transcriptome GRCm38-mm10.

Groups for Bioinformatics. Four groups were defined for differential expression analysis. Each group consisted of 6 mice ( 3 male, 3 female). Air $0 \mathrm{~h}$ mice were exposed to air and sacrificed immediately after their final session. Air $8 \mathrm{~h}$ mice were exposed to air and sacrificed 8 hours after their final session. Ethanol $0 \mathrm{~h}$ mice were exposed to ethanol and sacrificed immediately after their final session. Ethanol $8 \mathrm{~h}$ mice were exposed to ethanol and sacrificed 8 hours after their final session, while in withdrawal.

Differential Expression Analysis. Differential gene expression was calculated using DESeq2 ${ }^{26}$ (Galaxy Version 2.11.39). All Salmon and DESeq2 settings were left default. To determine microglia specific gene enrichment, all IP samples were compared to all Input samples. For all other test of differential expression, IP samples were compared to IP samples. To determine the effects of sacrifice time alone, Air $0 \mathrm{~h}$ mice were compared to Air $8 \mathrm{~h}$ mice; for the effects ethanol exposure, Ethanol oh mice were compared to all Air mice; for the effects of withdrawal, Ethanol $8 \mathrm{~h}$ mice were compared to Ethanol Oh mice. For all comparisons, a positive Wald statistic means that a gene is expressed more in the first group as compared to the second group. An FDR threshold of $q=0.05$ was used throughout the manuscript.

Gene Set Enrichment Analysis. Wald statics generated by DeSeq2 were used as the ranking variable for gene set enrichment analysis. All genes with reliable statistical comparisons (those not filtered by DeSeq2) were entered into WebGestalt $2019^{27}$ and GSEA was run all pertinent comparisons (enrichment, IP Air, IP 

available under aCC-BY-NC-ND 4.0 International license.
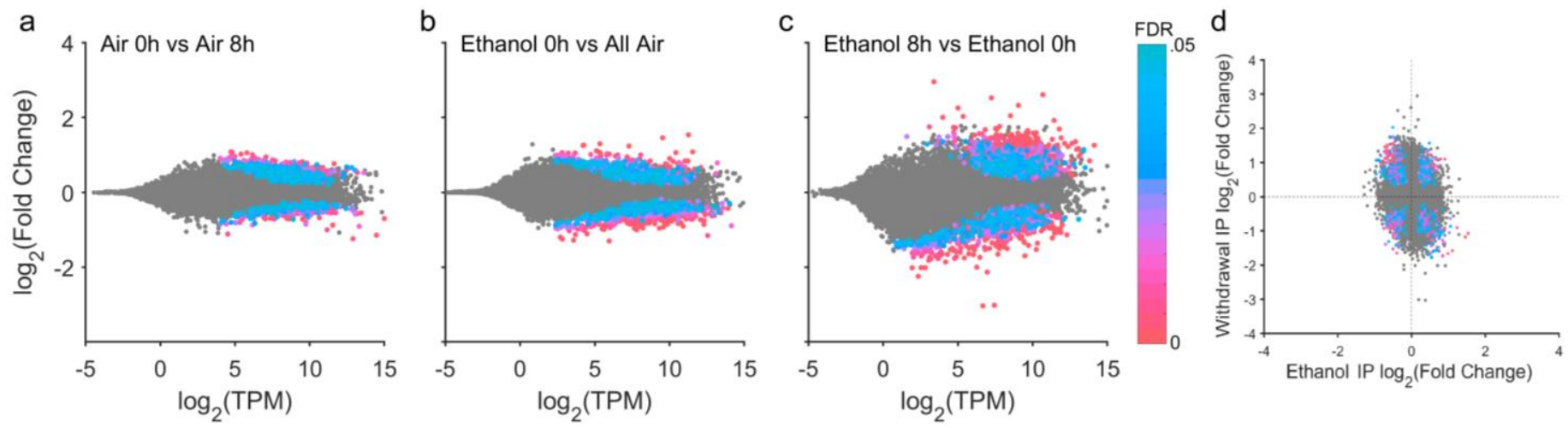

Figure 3. Differential Expression Analysis | a, Extraction time alone produced relatively few differentially expressed genes in air exposed controls. b, Ethanol exposure produced a roughly equal small number of upregulated and downregulated DEGs as compared to air exposed animals. c, Ethanol withdrawal produced dramatic differential gene expression in striatal microglia. d, Gene expression changes during withdrawal were not correlated to gene expression changes during ethanol exposure.

Ethanol, IP Withdrawal). Gene sets analyzed include GO: Biological Process, GO: Molecular Function, Keeg, Reactome, Wiki Pathways, Transcription Factor Targets, and MicroRNA Targets. All advanced parameters were left default except for significance level, which was set to FDR $=0.05$.

Weighted Gene Co-Expression Network Analysis. A topological overlap matrix for IP samples was generated, and module clustering was accomplished using the WGCNA ${ }^{28}$ package for $\mathrm{R}$ 29. Briefly, the TPM matrix for each group was filtered to remove zero-variance genes, and a signed adjacency matrix was generated using "bicor" as the correlation function. From this a signed topological overlap matrix was generated, followed by a dissimilarity topological overlap matrix. Finally, module membership was assigned using a dynamic tree cut. Clusters produced from this procedure are referred to as gene modules, and randomly renamed using the "Crayola Color Palette" in order to combat attribution of meaning or importance to numbered modules ${ }^{30}$. Modules were merged by clustering on module eigengenes with a cut height of 0.85 . A custom WGCNA MATLAB Class was used for graphing gene networks (GitHub.com/MxMarx/WGCNA).

Immunohistochemistry and Image Analysis for Iba1 Staining. Mice were deeply anesthetized with an intraperitoneal injection of sodium pentobarbital and phenytoin sodium and perfused transcardially with $100 \mathrm{ml}$ of $0.1 \mathrm{M}$ phosphate-buffered saline (PBS), followed by $250 \mathrm{ml}$ of cold $4 \%$ paraformaldehyde (pH 7.4). Brains were dissected, post fixed in $4 \%$ paraformaldehyde overnight at $4{ }^{\circ} \mathrm{C}$ and transferred to $30 \%$ sucrose in PBS. Sections $(40 \mu \mathrm{m})$ across the rostro-caudal axis of the striatum were collected on a sliding freezing microtome. For lba1 immunohistochemistry, free floating sections were rinsed in $1 X$ PBS ( $3 X, 10$ minutes), permeabilized with $0.5 \%$ Triton PBS, and blocked with $4 \%$ BSA in $0.3 \%$ Triton PBS at room temperature. Sections were incubated with primary anti-lba1 rabbit antibody diluted 1:500 (FUJIFILM Wako; \#019-19741) in 4\% BSA and 0.3\%
Triton PBS for $18 \mathrm{~h}$ at $4^{\circ} \mathrm{C}$. Sections were then rinsed with PBS ( $3 \mathrm{X}$, 10 minutes), incubated with 1: 400 dilution of Alexa Fluor 488 Goat anti-rabbit secondary antibody (ThermoFisher; \#A-11008) for sixty minutes at room temperature, washed with PBS (3X 10 minutes), mounted on charged slides, and cover slipped with ProLong Gold mounting medium (ThermoFisher; \#P36935).

Code and Data accessibility. Our bioinformatics analysis pipeline is archived on our Galaxy server ${ }^{31}$. All data code used to generate the figures in are available in our GitHub Repository ${ }^{32}$.

\section{Results}

Methodological Validations. Treating CX3CR1Cre ${ }^{\text {ERT2 }}$ | RiboTag ${ }^{\text {fl/ }}$ mice (Figure 1a) with tamoxifen resulted in strong expression of hemagglutinin (HA) tagged Rpl22 in lba1 expressing microglia (Figure 1c). Mice were then exposed to chronic intermittent ethanol vapor exposure (CIE, Figure $1 \mathrm{~b}$ ), consistently reaching blood ethanol concentrations of approximately $200 \mathrm{mg} / \mathrm{dL}$ (Supplementary Figure 1). RNA extraction from Input samples yielded $468 \pm 25 \mathrm{ng}$ (Mean \pm SEM) of RNA while IP samples yielded $59 \pm 6 \mathrm{ng}$ of RNA. Input samples were pooled within group (10ng per sample), and then 10ng of RNA from each sample was used for sequencing library preparation. RNA sequencing from IP samples yielded $6.83 \pm 0.18$ million reads (Mean $\pm S E M$ ) while Input samples yielded $3.58 \pm 1.00$ million reads. All fastq files passed FastQC basic statistics, per base quality and per sequence quality.

Microglia Specific Markers are Highly Enriched in RiboTag-Seq Samples. Differential expression analysis of IP vs Input samples isolated 2,418 genes significantly enriched in the IP samples. Among the highly enriched genes are numerous classic microglia markers such as Itgam (cd11b), Csf1r, Mpeg1, and CX3CR1 (Figure 2a). Gene set enrichment analysis (GSEA) of IP vs Input samples further validated our enrichment of classic microglia pathways, such as cytokine binding, purinergic receptor activity, and NF-kB binding (Figure $2 \mathrm{~b}$ ). This procedure is consistent across 
bioRxiv preprint doi: https://doi.org/10.1101/2020.09.02.280156; this version posted September 3, 2020. The copyright holder for this preprint (which was not certified by peer review) is the author/funder, who has granted bioRxiv a license to display the preprint in perpetuity. It is made available under aCC-BY-NC-ND 4.0 International license.
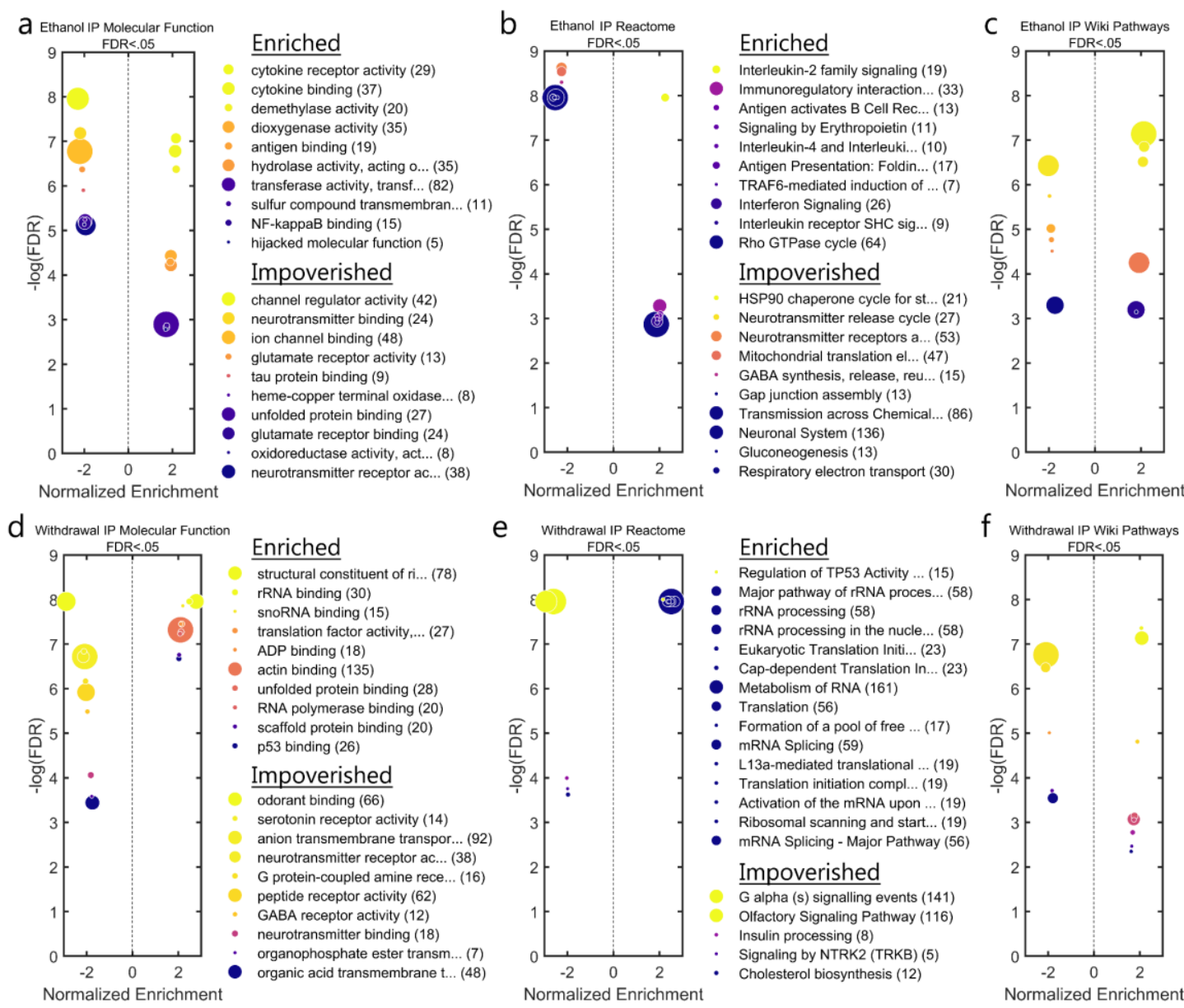

Enriched

Microglia Pathogen Phagocyto... (28)

TYROBP Causal Network (34)

Apoptosis (47)

Toll Like Receptor signaling (19)

Type II interferon signaling... (19)

- IL-5 Signaling Pathway (38)

- Kit Receptor Signaling Pathway (32)

NLR Proteins (6)

Impoverished

Glycogen Metabolism (17)

TCA Cycle (21)

Amino Acid metabolism (35)

Glycolysis and Gluconeogenesis (26)

Oxidative phosphorylation (35)

Electron Transport Chain (54)

Calcium Regulation in the Ca... (45)

Serotonin and anxiety (11)

Splicing factor NOVA regulat... (21)

Hypothetical Network for Dru... (14)

Serotonin and anxiety-relate... (10)

Normalized Enrichment

Normalized Enrichment

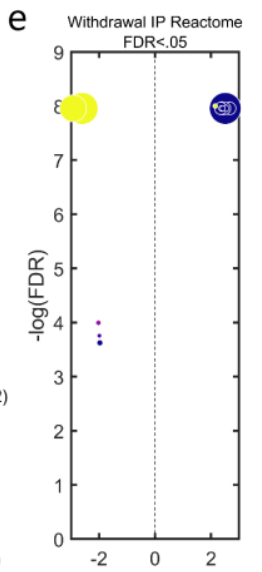

Enriched

f Myometrial Relaxation and Co... (38)

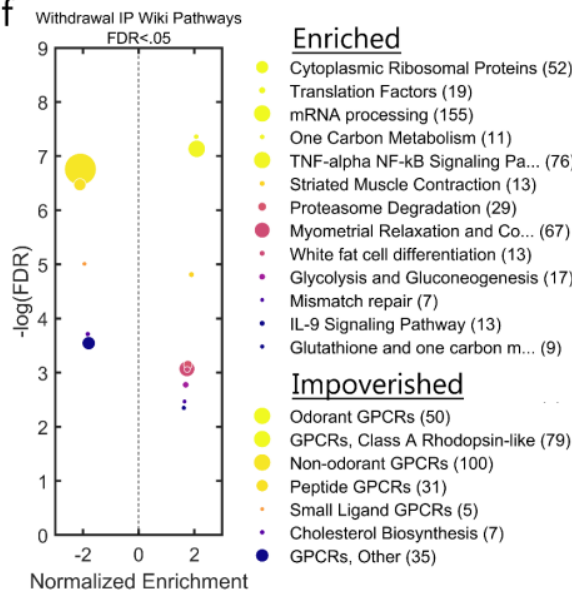

Figure 4. Gene Set Enrichment Analysis I GSEA reveals many annotated gene sets that are significantly upregulated or downregulated in microglia following ethanol intoxication using several databases, including Molecular Function (a), Reactome (b), and Wiki Pathways (c). For example, ethanol exposure upregulated classic microglial activation gene sets such as cytokine receptor binding, NF-kB binding, IL2, and IL4 signaling while the unfolded protein folding was downregulated. While some changes persisted, many gene sets changes were in the opposite direction, notably unfolded protein finding, using these databases, including Molecular Function (d), Reactome (e), and Wiki Pathways (f).

experiments; $80 \%$ of the differentially enriched genes in the present study were also differentially expressed in our previous CX3CR1-Ribotag study ${ }^{33}$ (Figure 2c). Further, genes that were highly enriched $(\log 2(\mathrm{fc})>4)$ shared nearly $100 \%$ concordance across experiments.

Ethanol Withdrawal Produce Dramatic Differential Gene Expression in Microglia. There were very few differentially expressed genes (DEGs) in microglia between the two Air exposure control groups (Air-0h vs Air-8h; Figure 3a). Ethanol exposure produced a modest 151 upregulated and 288 downregulated DEGs (Figure 3b). By contrast, withdrawal produced nearly double the number of DEGs, with 468 upregulated and 354 downregulated (Figure 3c). There was no consistent relationship in the direction or magnitude of change in DEGs when comparing ethanol exposure (EtOH-Oh vs all Air) to withdrawal (EtOH-8h vs. EtOH-Oh, Figure 3d).

Gene Set Enrichment Analysis of Microglia RNA During Ethanol Exposure and Withdrawal. GSEA provides many insights into how typical microglial function (i.e. phagocytosis, inflammatory signaling, etc.) was altered by ethanol and withdrawal; for brevity, the Wiki Pathways results are described here and others are presented in Figure 4. Relative to air treated controls, ethanol exposure (EtOH-Oh) resulted in an upregulation in five cytokine signaling gene sets, two phagocytosis gene sets, and the apoptosis gene set (Figure 4c). The upregulated cytokine signaling sets included Toll-Like Receptor (TLR) Signaling (NE: +2.13), Type II Interferon Signaling (NE: +2.08), Interleukin-5 (IL5) signaling (NE: +1.91), Nod-Like Receptor (NLR) proteins (NE: +1.79 ), and the Kit receptor signaling pathway (NE: +1.79), all indicative of increased neuroinflammation and microglial activation. Type II Interferon signaling were also regulated; and much like TLR4 pathways, IFN cascades are key top-down molecular regulators that activate a broad neuroinflammatory response ${ }^{34}$. The Microglial Pathogen Phagocytosis Network (NE: +2.37) and the TYROBP network (NE: +2.35) ${ }^{35}$, which are associated with active phagocytosis, were also upregulated by ethanol exposure. The Apoptosis gene set (NE: +2.12) was also 

available under aCC-BY-NC-ND 4.0 International license.

upregulated, suggesting that there was an increase in microglial cell death. Ethanol exposure was also associated with a downregulation in six metabolic gene sets, including the Glycogen Metabolism (NE: -2.25), Glycolysis and Gluconeogenesis (NE: -2.80), TCA Cycle (NE: -2.26), Amino Acid Metabolism (NE: -2.29), Oxidative Phosphorylation (NE: -2.88), and Electron Transport Chain (NE: -2.93) sets. While this likely reflects ethanol's well-known interference with metabolic pathways ${ }^{36}$, it also suggests that ethanol induced metabolic impairment may negatively impact the functional integrity of microglia.

Relative to the acute ethanol intoxication timepoint (EtOHOh), numerous gene sets were exclusively altered during withdrawal (EtOH-8h, Figure 4f). There was an upregulation in the pro-inflammatory TNF $\alpha / N F k B$ cytokine gene set (NE: +2.12 ), as well as an upregulation in numerous mRNA translation associated gene sets including the Cytoplasmic Ribosomal Proteins set (NE: +2.77$)$, the mRNA processing set (NE: +2.15), and the Translation Factors set (NE: +2.22). There was also an upregulation in cellular metabolism associated gene sets including the One Carbon Metabolism set (NE: +2.06) and the Proteasome Degradation set (NE: +1.77). Finally, numerous GPCR gene sets were downregulated during withdrawal, including the Class A Rhodopsin Like GPCRs (NE: -2.41), Odorant (NE: -2.35) and Non-Odorant GPCRs (NE: -2.10), Peptide GPCRs (NE: -2.11), Small Ligand GPCRs (NE: -1.95), and "Other" GPCR (NE: -1.80) gene sets. In addition, some gene sets changed in opposite directions in the $\mathrm{EtOH}-\mathrm{Oh}$ vs. Air and the EtOH-8h vs EtOH-Oh; for example, the Glycolysis and Gluconeogenesis set was decreased during intoxication but increased during withdrawal (Figure $4 \mathrm{c}$ and $\mathrm{f}$, respectively).

WGCNA Reveals the Induction of the Unfolded Protein Response in Striatal Microglia during Ethanol Withdrawal. A minimum spanning tree of the entire RiboTag-Seq matrix and corresponding module labels were projected into $2 \mathrm{D}$ space using a force directed layout (Figure 5a). Significance values for ethanol exposure (Figure 5b) and withdrawal (Figure 5c) are overlaid on the minimum spanning tree to visualize the relationship between modules and individual gene significance. Functionally related genes are generally sorted into distinct modules by the WGCNA process, even though the clustering is not guided by any a priori considerations about gene function. In numerous cases, highly significant DEGs fell into different gene modules in the ethanol exposure and withdrawal conditions, suggesting that there was mostly independent regulation of gene networks.

Of particular interest are modules that were differentially expressed between treatment groups (Supplementary Figure 1). One example, (Orange-Yellow) was examined to determine if network membership was meaningful or just capturing a simple
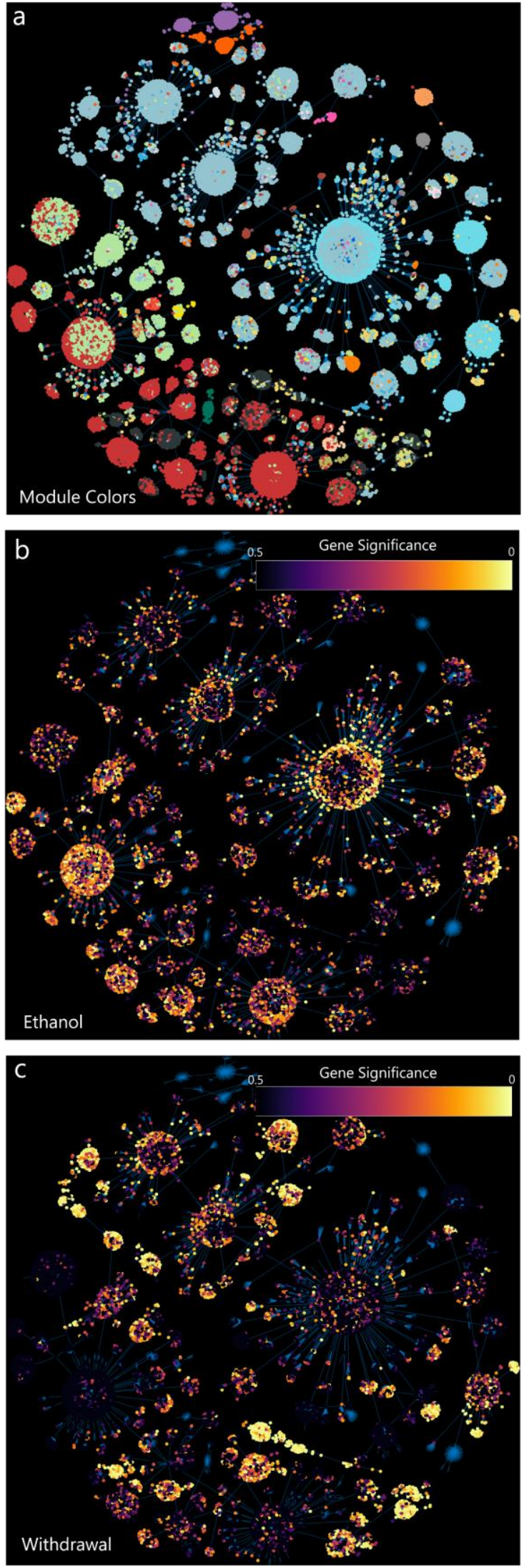

Figure 5. WGCNA Minimum Spanning Tree | a, Genes are coded by module color and projected into a 2D minimum spanning tree using a "force" layout. b, The same genes are coded by differential expression (q-value) data during ethanol exposure. c, The same genes are coded by differential expression (q-value) data during ethanol withdrawal. Functionally related genes tend to cluster. 

available under aCC-BY-NC-ND 4.0 International license.

inverse relationship in differential expression. An undirected network graph was generated for the Orange-Yellow module and was projected onto 2D space using a circle layout (Figure 6a) and a minimum spanning tree projection (Figure 6b). Each gene's importance within the network is defined using a centrality metric, calculated using "closeness" for the distance calculation, and the sum of each node's edge weight (similarity) as the "cost" function. Genes that are central to the network form many strong connections with other genes, which is represented by many bright lines connecting a gene to others in the network graphs. For the Orange-Yellow module, network centrality was highly correlated to individual gene differential expression during withdrawal (Figure 6c). Over-representation analysis (ORA) was used to determine if the genes in the Orange-Yellow module likely originated from any known gene pathways (Figure 6d). ORA was performed using WebGestalt ${ }^{27}$ to search Biological Process, Molecular Function, KEGG, Reactome, and Transcription Factor Target gene sets. One key pathway present in the Orange-Yellow network are genes engaged in the unfolded protein response (UPR, Figure 6d). This is consistent with the Molecular Pathway GSEA results that indicated a downregulation in the unfolded protein binding set during intoxication, followed by an upregulation during withdrawal (Figure $4 a$ and $d$, respectively). The UPR is activated in response to an accumulation of unfolded or misfolded proteins in the lumen of the endoplasmic reticulum (ER) and is a highly conserved cellular stress response related to ER stress. The UPR can be a homeostatic response to return the system to baseline but may also lead to stress-induced apoptotic cell death. There are three major ER sensors that can initiate this system: PERK (Eif2Ak3), ATF4, and IER1 (Ern1). IER1 was significantly increased in Ethanol-Oh mice compared to Air controls and is associated with early time points of UPR activation, whereas ATF4 is considered a later phase of activation 37 and was increased in Ethanol-8h animals during withdrawal. Since ATF4 is a key activator of the transcription factor C/EBP homologous protein (CHOP, gene symbol Ddit3), we examined the level of ribosome associated Ddit3 mRNA and found that it was significantly increased by withdrawal $(q=0.002)$. Other gene modules may also be of interest but were not analyzed deeply in this report and can be reviewed in the code repository.

\section{Discussion}

The main results of this study showed that there were dramatic changes in RNA actively undergoing translation (the "translatome") in striatal microglia of mice that were exposed to repeated cycles of ethanol vapor exposure and withdrawal. Using deep sequencing of RiboTag-enriched RNA from microglia, we used three strategies to analyze the effect of chronic ethanol exposure and withdrawal on differential expression within the microglial translatome.
The RiboTag procedure yielded a dramatic enrichment of gene associated with microglia as compared to total RNA in the striatum (Figure 2). One advantage of the method used here is that the RiboTag procedure provides a snapshot of RNA actively undergoing translation at the time of brain extraction and is derived from all ribosomes in the cell type expressing the RiboTag protein. Thus, there is no loss of RNA due to cell fractionation and sorting, preserving RNA from ribosomes throughout the cell cytoplasm and no change in RNA expression due to altered gene expression induced by the cell sorting procedure. Furthermore, we reasoned that RNAs that were translated together might also be co-regulated by the experimental manipulations, providing new insights into functional networks of genes within microglia during ethanol dependence and withdrawal. Accordingly, while our results are consistent with other studies investigating glialassociated $\mathrm{CIE}$ induced alterations in gene expression using alternative methods ${ }^{38,39}$, we believe that RiboTag enabled us to extend the findings of these previous studies by identifying additional novel alcohol sensitive genes networks.

We first used DESeq2 and found that there were many changes in individual gene expression levels between ethanol treated animals and air controls, and between ethanol exposed animals at 0 hours (intoxicated) vs. 8 hours (withdrawal) after discontinuation of ethanol vapor exposure. Hundreds of genes were either up or downregulated between treatment conditions (Figure 3). These individual changes provide a great deal of data and potential leads for subsequent analysis and are presented in the supplemental data.

We next used GSEA to produce a more integrative analysis of the gene changes. GSEA is a method that used previously curated sets of functionally defined genes and examines whether there is a statistical over-representation of genes associated with these gene sets in any of the experimentally manipulated treatment groups. Using this method, we found a pattern of changes that suggested the induction of inflammatory and cytokine signaling in microglia after repeated cycles of ethanol exposure (EtOH-Oh), as well as an upregulation in the TNF $\alpha$ cytokine gene set exclusively at 8 hours of withdrawal from ethanol vapor, a timepoint that has previously been associated with intense biological and behavioral signs of ethanol withdrawal ${ }^{40}$. Although GSEA is a very sensitive strategy for detecting meaningful patterns of gene changes, it is based on the observations that were used to curate the gene sets in the first place. However, we observed similar patterns of changes across multiple databases, suggesting that the biological pathway perturbations indicated by the GSEA analysis are informative.

The third analytic strategy that we used, WCGNA, is an unbiased clustering strategy that detects the relatedness amongst all of the genes measured across all of the individuals 

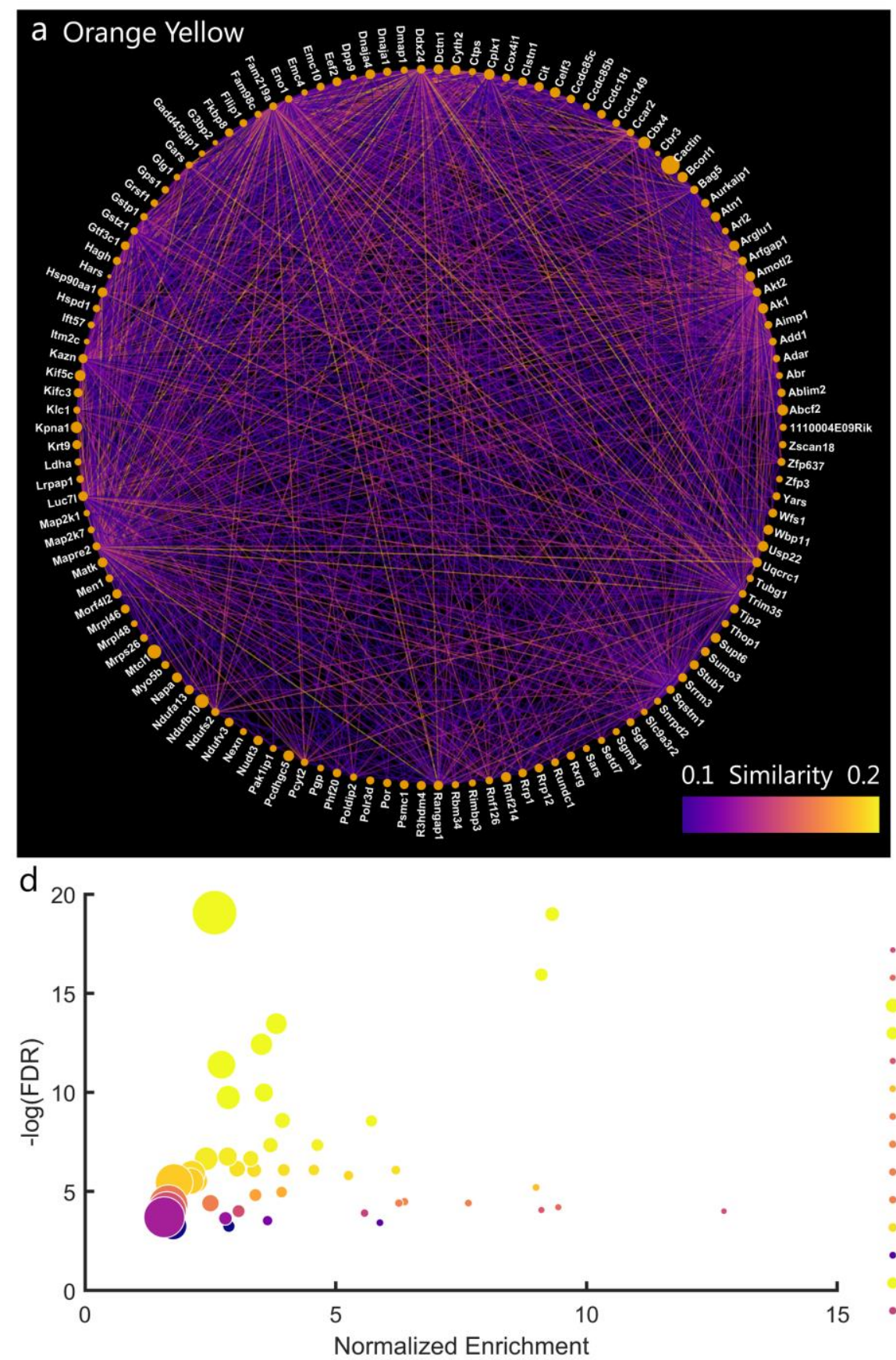
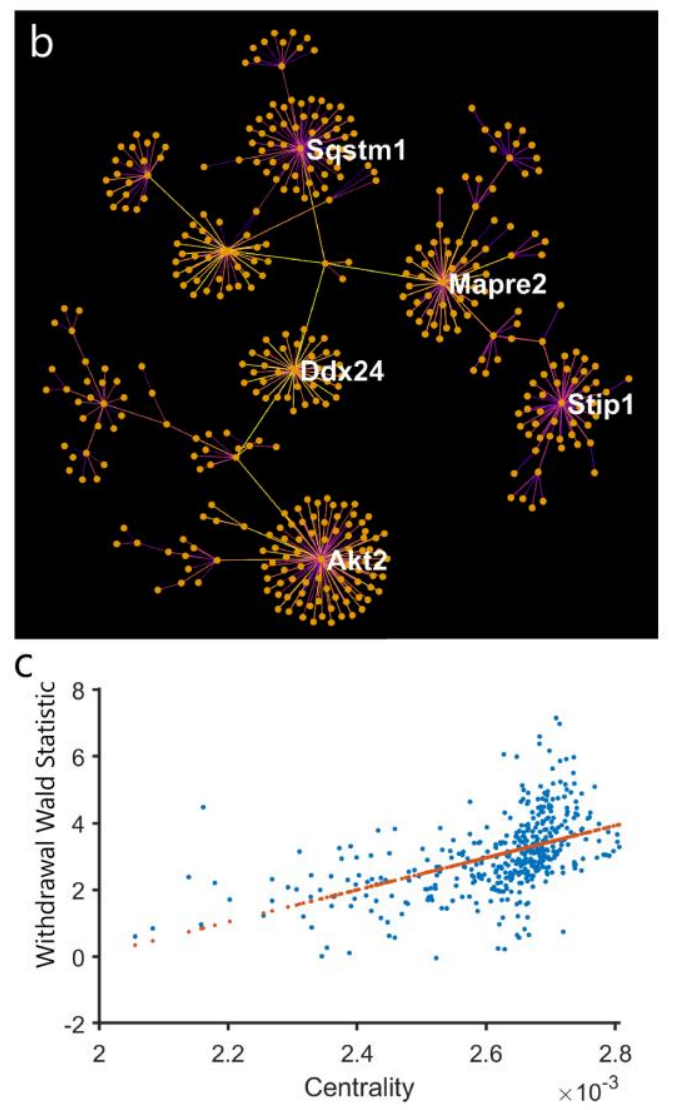

Gene Set
response to interleukin-7 (4)
chaperone cofactor-dependent protein ... (5)
unfolded protein binding (17)
Mitochondrial translation elongation (15)
positive regulation of telomere maint... (5)
regulation of transcription elongatio... (6)
tRNA aminoacylation for protein trans... (6)
mitochondrial ATP synthesis coupled e... (7)
protein transmembrane transport (7)
Complex I biogenesis (7)
telomere maintenance via telomere len... (9)
SCF-beta-TrCP mediated degradation of... (6)
response to heat (13)
Regulation of HSF1-mediated heat shoc... (7)

Figure 6. Orange Module Network Analysis | a, The Orange Yellow module projected into 2D space using a circle layout. Line brightness represents gene similarity as calculated by WGCNA. b. Minimum spanning tree of the Orange Yellow module showing the top five most central genes. c, Centrality (a measure of network importance) in the Orange Yellow network is correlated to differential expression for withdrawal. $\mathbf{d}$, Over-representation analysis on the Orange Yellow module shows that genes related to IL-7 response, the unfolded protein response (UPR) and others are upregulated during ethanol withdrawal in striatal microglia.

sampled in the study. This approach complements the other two strategies but may be useful in identifying novel patterns of gene changes and potentially novel genes that have not been functionally defined as of yet. WCGNA of this dataset revealed robust modules of genes which had similar expression patterns and there may be many insights to be gleaned from examining several of these modules. The "Orange-Yellow" module was particularly intriguing as it was differentially regulated in ethanol intoxication vs. withdrawal. Using the DEG analysis generated by DESeq2 (Figure 3) we found that the Wald score for each gene in this module was strongly correlated with its centrality in the Orange-Yellow module, suggesting that this module was meaningfully associated with key biological processes that were in response to ethanol withdrawal. It is possible that both mediators and mitigators of withdrawal were identified. The full list of genes in this module reflect a range of genes that encode 

available under aCC-BY-NC-ND 4.0 International license.

both well-known and novel proteins (Figure 6a) and the most central genes of this module reflect the coregulation of the entire module. These most central genes have been framed as "hub" genes in other studies ${ }^{41}$ although they may or may not directly regulate the other genes with which they are correlated. In order to further interpret this gene module, we performed overrepresentation analysis on this gene set, which is analogous to GSEA and we found that, among these were gene sets relating to the unfolded protein response (UPR). This result is intriguing because the UPR has been associated with ethanol toxicity and withdrawal in other tissues ${ }^{42}$ and other cell types including macrophages ${ }^{43}$ but has not been identified in microglia in the central nervous system. Interestingly, Erickson and colleagues detected altered expression of a module involving response to unfolded protein in both cortical microglia and astrocytes in acutely intoxicated mice after CIE whereas we observed this module to be reduced in $\mathrm{EtOH}-\mathrm{Oh}$ animals compared to Air controls but increased after withdrawal ${ }^{38}$.

The UPR is a cellular response to stress that can be initiated when there is increased detection of misfolded proteins in the endoplasmic reticulum or mitochondria; the UPR can be a homeostatic response to return the system to baseline but may also lead to stress-induced apoptotic cell death. There are three major ER sensors that can initiate this system: PERK (Eif2ak3), ATF6, and IER1 (Ern1). IER1 was significantly increased in EtOH-0 mice compared to Air controls and is associated with early time points of UPR activation, whereas ATF4 is considered a later phase of activation ${ }^{37}$ and was increased in $\mathrm{EtOH}-8 \mathrm{~h}$ animals. Since ATF4 is a key activator of the transcription factor C/EBP homologous protein (CHOP, gene symbol Ddit3, in the orangeyellow module), we examined the level of ribosome associated Ddit3 mRNA and found that it was elevated in EtOH-8h compared to EtOH-Oh. Since Ddit3 RNA translation was increased only in the EtOH-8h group, it suggests that this may be a downstream development during ethanol withdrawal. This may have important clinical implications as that induction of $\mathrm{CHOP}$ is associated with the induction of apoptosis ${ }^{44}$. UPR and $\mathrm{CHOP}$ have been implicated in inflammatory processes associated with neurodegenerative disease models ${ }^{45,46}$, and after cocaineinduced microglial activation ${ }^{47}$. The potential role of $\mathrm{CHOP}$ in microglia after ethanol exposure has received little attention, although it's induction in peripheral tissues ${ }^{48}$ and in brain ${ }^{49}$ has been well documented. In one case where ethanol increased CNS $\mathrm{CHOP}$, it was found in neurons but not astroglia, although microglia were not studied ${ }^{49}$.

One limitation of the present study is that it precluded simultaneous immunohistochemical confirmation because the RiboTag associated RNA was prepared from unfixed fresh tissue. We did confirm some of the target RNAs using RTqPCR, but there was limited RNA available after preparation of the sequencing libraries. Future studies should investigate role of the UPR in microglia following chronic ethanol exposure and withdrawal.

Neuroinflammation, including microglial mediated inflammatory responses, have been identified as important regulators of the behavioral and neuropathological consequences of repeated cycles of alcohol intoxication and withdrawal. However, the mechanisms are complex, and it is hard to distinguish whether microglial markers associated with these processes reflect an alcohol induced impairment in healthy microglial function versus an adaptive microglial response to the pathological effects of alcohol on other neuronal cell types or tissues. It is likely that both occur. Here, by using RiboTag in combination with deep sequencing and bioinformatics to selectively assess microglial specific transcriptional alterations, we more fully characterize the microglial response to chronic ethanol exposure at timepoints reflecting acute intoxication and withdrawal, providing insights into these possibly divergent (pathological vs adaptive) processes. In particular, we identified alcohol-associated impairments in microglial function, including a downregulation in the UPR cellular stress response and impaired metabolic function. We found robust indicators of microglial activation, including an inflammatory cytokine response that evolves over the withdrawal timeline, as well as upregulated markers of active phagocytosis and apoptosis.

By identifying affected gene networks and specific individual genes associated with acute intoxication and withdrawal in an alcohol dependence model, we have identified multiple novel points of possible therapeutic intervention. In particular, therapeutics that rescue alcohol induced impairments in microglial UPR and metabolism could improve the adaptive response of microglia to alcohol exposure. Similarly, therapeutic modulation of microglial could possibly help to attenuate the deleterious proinflammatory cytokine associated neurotoxicity characteristic of withdrawal. Ideally, either approach could be utilized to possibly reduced the deleterious consequences of chronic alcohol use and withdrawal symptomology, and thereby warrants further investigation. 


\section{References}

1 (SAMHSA), S. A. a. M. H. S. A. 2018 National Survey on Drug Use and Health (NSDUH)

$<$ https://www.samhsa.gov/data/sites/default/files/cbhsqreports/NSDUHDetailedTabs2018R2/NSDUHDetTabsSect5pe2018.htm\#tab5-4a> (2018).

Sacks, J. J., Gonzales, K. R., Bouchery, E. E., Tomedi, L. E. \& Brewer, R. D. 2010 National and State Costs of Excessive Alcohol Consumption. Am J Prev Med 49, e73-e79, doi:10.1016/j.amepre.2015.05.031 (2015).

Schuckit, M. A. Recognition and management of withdrawal delirium (delirium tremens). N Engl J Med 371, 2109-2113, doi:10.1056/NEJMra1407298 (2014).

Victor, M. \& Brausch, C. The role of abstinence in the genesis of alcoholic epilepsy. Epilepsia 8, 1-20, doi:10.1111/j.1528-1157.1967.tb03815.x (1967).

Campos, J., Roca, L., Gude, F. \& Gonzalez-Quintela, A. Long-term mortality of patients admitted to the hospital with alcohol withdrawal syndrome. Alcohol Clin Exp Res 35, 1180-1186, doi:10.1111/j.1530-0277.2011.01451.x (2011).

Heilig, M., Egli, M., Crabbe, J. C. \& Becker, H. C. Acute withdrawal, protracted abstinence and negative affect in alcoholism: are they linked? Addict Biol 15, 169-184, doi:10.1111/j.1369-1600.2009.00194.x (2010).

Koob, G. F. Neurobiology of Opioid Addiction: Opponent Process, Hyperkatifeia, and Negative Reinforcement. Biol. Psychiatry 87, 44-53, doi:10.1016/j.biopsych.2019.05.023 (2020).

Crews, F. T., Lawrimore, C. J., Walter, T. J. \& Coleman, L. G., Jr. The role of neuroimmune signaling in alcoholism. Neuropharmacology 122, 56-73, doi:10.1016/j.neuropharm.2017.01.031 (2017).

Qin, L. \& Crews, F. T. NADPH oxidase and reactive oxygen species contribute to alcohol-induced microglial activation and neurodegeneration. J Neuroinflammation 9, 5, doi:10.1186/1742-2094-9-5 (2012).

Gilman, S. et al. Positron emission tomographic studies of cerebral benzodiazepine-receptor binding in chronic alcoholics. Ann Neurol 40, 163-171, doi:10.1002/ana.410400207 (1996).

11 Tsai, G., Gastfriend, D. R. \& Coyle, J. T. The glutamatergic basis of human alcoholism. Am J Psychiatry 152, 332340, doi:10.1176/ajp.152.3.332 (1995).

12 Harding, A. J., Halliday, G. M., Ng, J. L., Harper, C. G. \& Kril, J. J. Loss of vasopressin-immunoreactive neurons in alcoholics is dose-related and time-dependent. Neuroscience 72, 699-708, doi:10.1016/0306-4522(95)00577-3 (1996).

13 Miguel-Hidalgo, J. J., Overholser, J. C., Meltzer, H. Y., Stockmeier, C. A. \& Rajkowska, G. Reduced glial and neuronal packing density in the orbitofrontal cortex in alcohol dependence and its relationship with suicide and duration of alcohol dependence. Alcohol Clin Exp Res 30, 1845-1855, doi:10.1111/j.1530-0277.2006.00221.x (2006).

14 Becker, H. C. \& Hale, R. L. Repeated episodes of ethanol withdrawal potentiate the severity of subsequent withdrawal seizures: an animal model of alcohol withdrawal "kindling". Alcohol Clin Exp Res 17, 94-98, doi:10.1111/j.1530-0277.1993.tb00731.x (1993).

15 He, J. \& Crews, F. T. Increased MCP-1 and microglia in various regions of the human alcoholic brain. Exp Neurol 210, 349-358, doi:10.1016/j.expneurol.2007.11.017 (2008).

Badanich, K. A., Becker, H. C. \& Woodward, J. J. Effects of chronic intermittent ethanol exposure on orbitofrontal and medial prefrontal cortex-dependent behaviors in mice. Behav Neurosci 125, 879-891, doi:10.1037/a0025922 (2011).

17 Kettenmann, H., Hanisch, U. K., Noda, M. \& Verkhratsky, A. Physiology of microglia. Physiol Rev 91, 461-553, doi:10.1152/physrev.00011.2010 (2011).

Hanisch, U. K. Microglia as a source and target of cytokines. Glia 40, 140-155, doi:10.1002/glia.10161 (2002).

Salter, M. W. \& Stevens, B. Microglia emerge as central players in brain disease. Nat. Med. 23, 1018-1027, doi:10.1038/nm.4397 (2017). 
20 National Research Council. Guide for the Care and Use of Laboratory Animals: Eighth Edition. (The National Academies Press, 2011).

21 Rose, J. H. et al. Supersensitive Kappa Opioid Receptors Promotes Ethanol Withdrawal-Related Behaviors and Reduce Dopamine Signaling in the Nucleus Accumbens. Int. J. Neuropsychopharmacol. 19, doi:10.1093/ijnp/pyv127 (2016).

Morton, R. A., Diaz, M. R., Topper, L. A. \& Valenzuela, C. F. Construction of vapor chambers used to expose mice to alcohol during the equivalent of all three trimesters of human development. J Vis Exp, doi:10.3791/51839 (2014).

Lesiak, A. J. et al. Sequencing the serotonergic neuron translatome reveals a new role for Fkbp5 in stress. Mol. Psychiatry, doi:10.1038/s41380-020-0750-4 (2020).

24 Afgan, E. et al. The Galaxy platform for accessible, reproducible and collaborative biomedical analyses: 2016 update. Nucleic Acids Res. 44, W3-W10, doi:10.1093/nar/gkw343 (2016).

Patro, R., Duggal, G., Love, M. I., Irizarry, R. A. \& Kingsford, C. Salmon provides fast and bias-aware quantification of transcript expression. Nature methods 14, 417-419, doi:10.1038/nmeth.4197 (2017). DESeq2. Genome Biol. 15, 550, doi:10.1186/s13059-014-0550-8 (2014).

Liao, Y., Wang, J., Jaehnig, E. J., Shi, Z. \& Zhang, B. WebGestalt 2019: gene set analysis toolkit with revamped Uls and APIs. Nucleic Acids Res. 47, W199-W205, doi:10.1093/nar/gkz401 (2019).

Langfelder, P. \& Horvath, S. WGCNA: an R package for weighted correlation network analysis. BMC Bioinformatics 9, 559, doi:10.1186/1471-2105-9-559 (2008).

Cobeldick, S. Convert between RGB and Color Names, <https://www.mathworks.com/matlabcentral/fileexchange/48155-convert-between-rgb-and-color-names> (2020).

31 Coffey, K. R. http://128.95.144.9:8080/u/kcgalaxy/h/imported-ethanol-microglia.

Coffey, K. R. https://github.com/DrCoffey/Manuscripts/tree/master/Ethanol\%20Microglia\%20(unpublished).

33 Coffey, K. R. et al. RiboTag-Seq Reveals a Compensatory cAMP Responsive Gene Network in Striatal Microglia Induced by Morphine Withdrawal. bioRxiv (2020).

Monteiro, S., Roque, S., Marques, F., Correia-Neves, M. \& Cerqueira, J. J. Brain interference: Revisiting the role of IFNgamma in the central nervous system. Prog Neurobiol 156, 149-163, doi:10.1016/j.pneurobio.2017.05.003 (2017).

35 Konishi, H. \& Kiyama, H. Microglial TREM2/DAP12 Signaling: A Double-Edged Sword in Neural Diseases. Front Cell Neurosci 12, 206, doi:10.3389/fncel.2018.00206 (2018).

Manzo-Avalos, S. \& Saavedra-Molina, A. Cellular and mitochondrial effects of alcohol consumption. Int J Environ Res Public Health 7, 4281-4304, doi:10.3390/ijerph7124281 (2010).

37 Nishitoh, H. CHOP is a multifunctional transcription factor in the ER stress response. J Biochem 151, 217-219, doi:10.1093/jb/mvr143 (2012).

38 Erickson, E. K., Blednov, Y. A., Harris, R. A. \& Mayfield, R. D. Glial gene networks associated with alcohol dependence. Sci Rep 9, 10949, doi:10.1038/s41598-019-47454-4 (2019). in gene coexpression networks. PLoS One 10, e0121522, doi:10.1371/journal.pone.0121522 (2015).

41 Lorsch, Z. S. et al. Stress resilience is promoted by a Zfp189-driven transcriptional network in prefrontal cortex. Nat Neurosci 22, 1413-1423, doi:10.1038/s41593-019-0462-8 (2019). 
42 Kaplowitz, N. \& Ji, C. Unfolding new mechanisms of alcoholic liver disease in the endoplasmic reticulum. J Gastroenterol Hepatol 21 Suppl 3, S7-9, doi:10.1111/j.1440-1746.2006.04581.x (2006).

43 Kaphalia, L., Srinivasan, M. P., Kakumanu, R. D., Kaphalia, B. S. \& Calhoun, W. J. Ethanol Exposure Impairs AMPK Signaling and Phagocytosis in Human Alveolar Macrophages: Role of Ethanol Metabolism. Alcohol Clin Exp Res 43, 1682-1694, doi:10.1111/acer.14131 (2019).

44 Li, Y., Guo, Y., Tang, J., Jiang, J. \& Chen, Z. New insights into the roles of CHOP-induced apoptosis in ER stress. Acta Biochim Biophys Sin (Shanghai) 46, 629-640, doi:10.1093/abbs/gmu048 (2014).

45 Kamarehei, M. et al. Increased expression of endoplasmic reticulum stress-related caspase-12 and CHOP in the hippocampus of EAE mice. Brain Res Bull 147, 174-182, doi:10.1016/j.brainresbull.2019.01.020 (2019).

46 Mhaille, A. N. et al. Increased expression of endoplasmic reticulum stress-related signaling pathway molecules in multiple sclerosis lesions. J Neuropathol Exp Neurol 67, 200-211, doi:10.1097/NEN.0b013e318165b239 (2008).

47 Guo, M. L. et al. Cocaine-mediated microglial activation involves the ER stress-autophagy axis. Autophagy 11, 995-1009, doi:10.1080/15548627.2015.1052205 (2015).

$48 \mathrm{Ji}, \mathrm{C} ., \mathrm{M}$ Mehrian-Shai, R., Chan, C., Hsu, Y. H. \& Kaplowitz, N. Role of CHOP in hepatic apoptosis in the murine model of intragastric ethanol feeding. Alcohol Clin Exp Res 29, 1496-1503, doi:10.1097/01.alc.0000174691.03751.11 (2005).

49 Ke, Z. et al. Ethanol induces endoplasmic reticulum stress in the developing brain. Alcohol Clin Exp Res 35, 15741583, doi:10.1111/j.1530-0277.2011.01503.x (2011). 
bioRxiv preprint doi: https://doi.org/10.1101/2020.09.02.280156; this version posted September 3, 2020. The copyright holder for this preprint (which was not certified by peer review) is the author/funder, who has granted bioRxiv a license to display the preprint in perpetuity. It is made available under aCC-BY-NC-ND 4.0 International license.

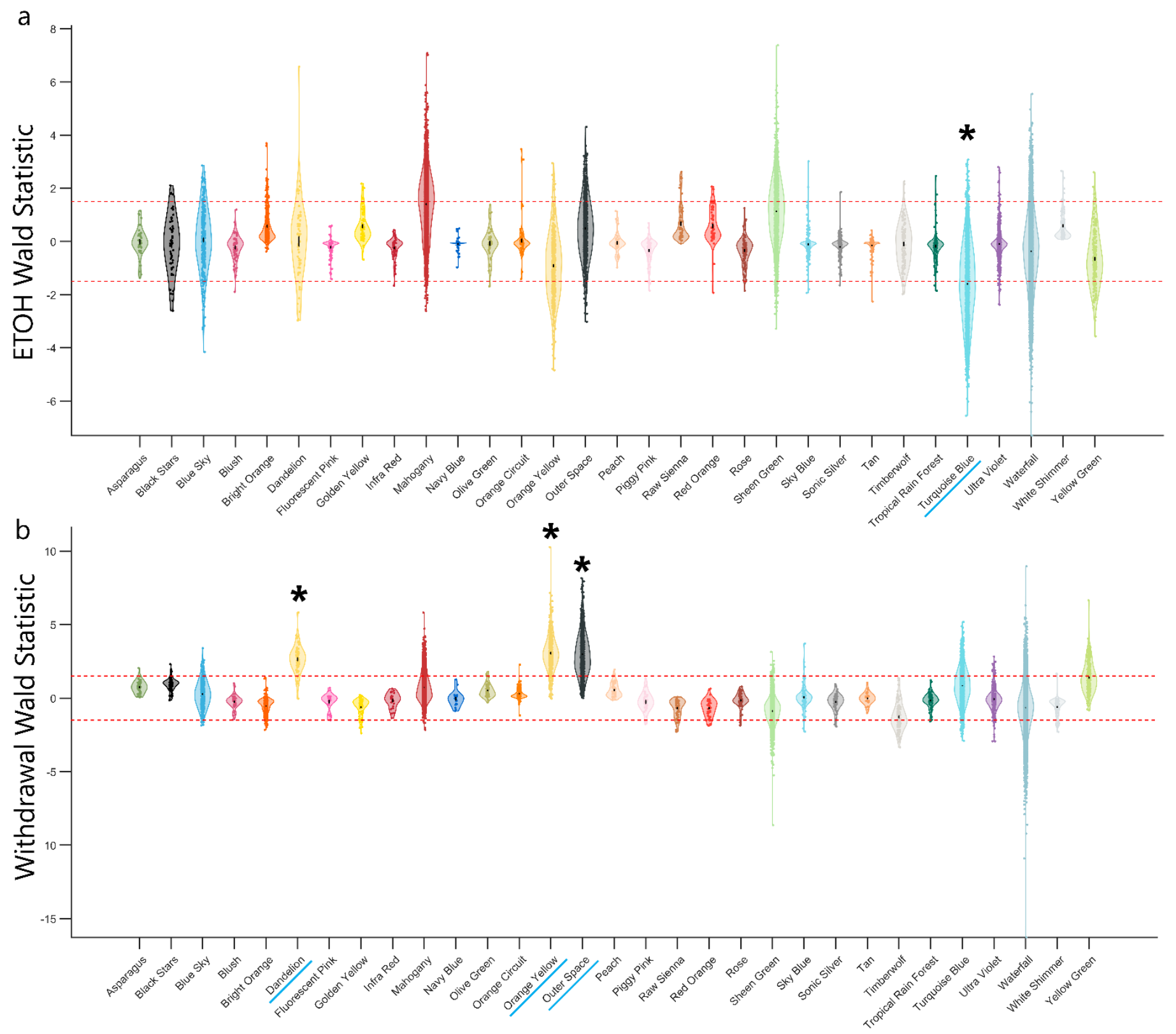

Supplementary Figure 1. WGCNA Module Significance | Most gene modules are unrelated to ethanol exposure or withdrawal. a, A single gene module, turquoise blue, was on-average down regulated by ethanol, and $\mathbf{b}$, three modules, dandelion, orange-yellow, and outer space, were on-average upregulated by withdrawal. 
bioRxiv preprint doi: https://doi.org/10.1101/2020.09.02.280156; this version posted September 3, 2020. The copyright holder for this preprint (which was not certified by peer review) is the author/funder, who has granted bioRxiv a license to display the preprint in perpetuity. It is made available under aCC-BY-NC-ND 4.0 International license.

\section{a Ethanol Vapor Chamber Dose Response}

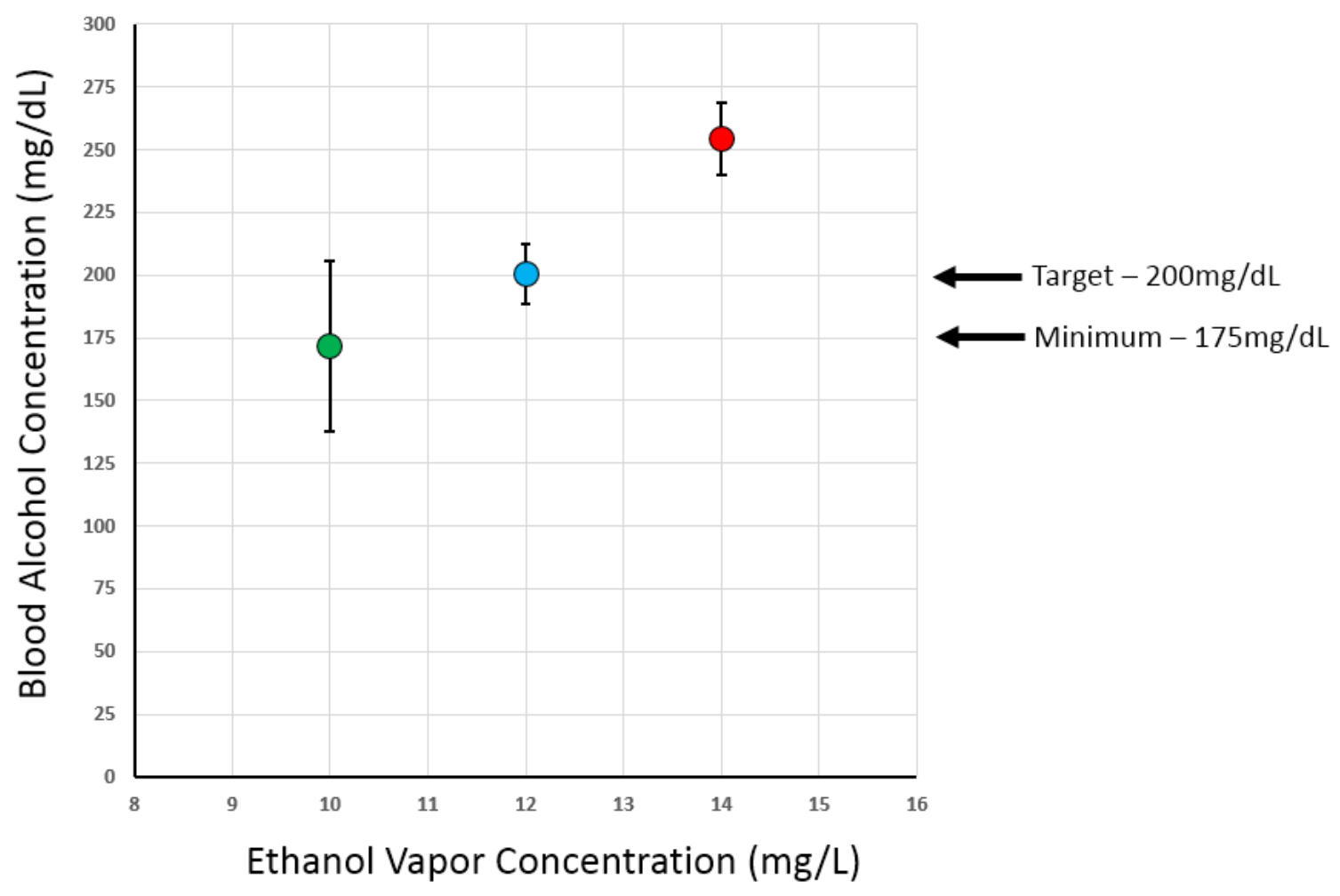

\section{b Blood Alcohol Stability (4 and 10 hours)}

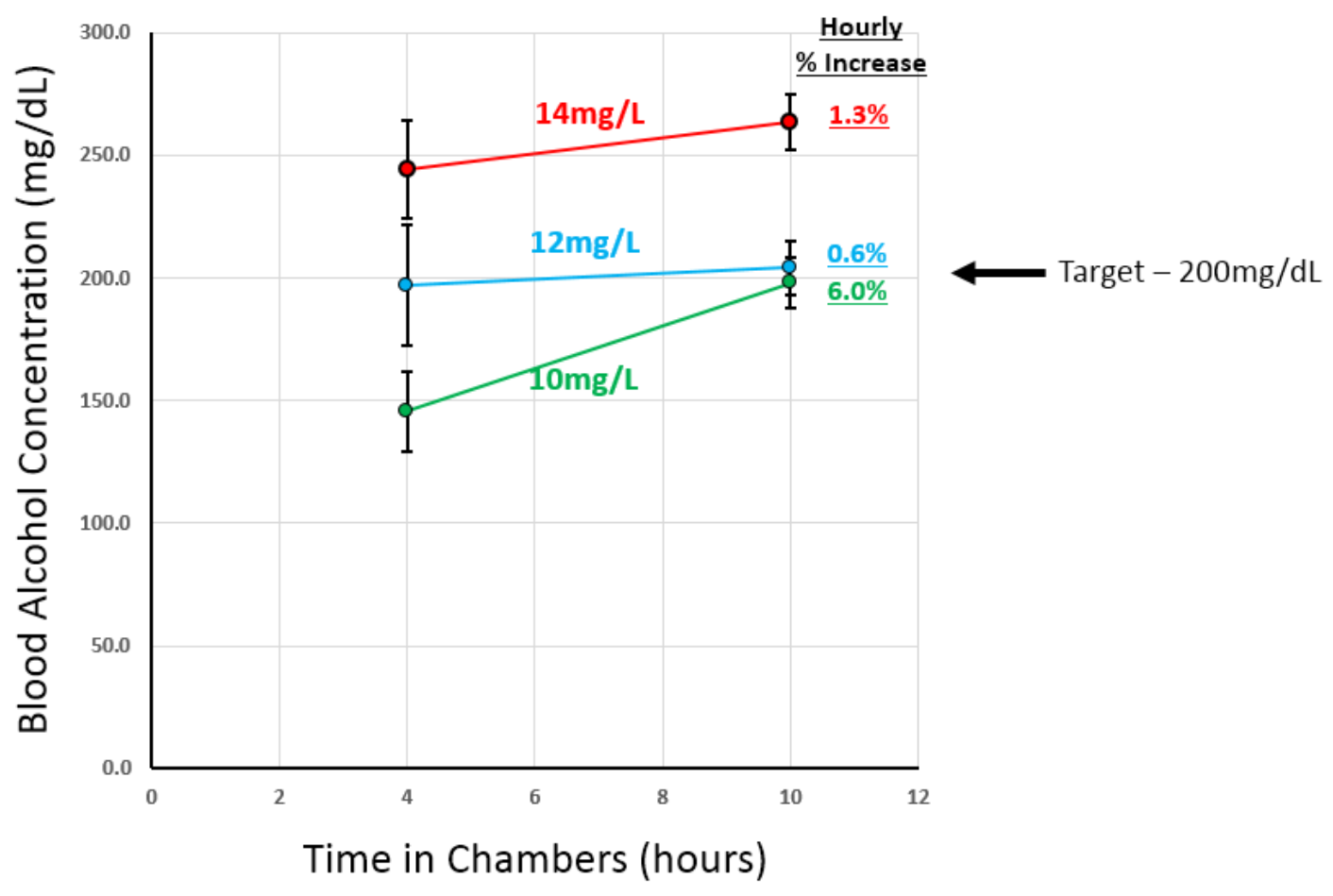

Supplementary Figure 2. Blood Alcohol Stability| a, Blood alcohol concentration is closely related to ethanol vapor concentration allowing us to tightly control blood alcohol concentration around $200 \mathrm{mg} / \mathrm{dL}$. b, At $12 \mathrm{mg} / \mathrm{L}$ ethanol vapor concentration, blood alcohol concentration remains steady across all 10 hours in the chamber. 